\title{
¿Quién responde por la formación de los docentes como sujetos de derechos en las facultades de educación? ${ }^{1}$
}

\author{
Who is Responsible for Future Teachers' Training \\ on Human Rights in the Faculties of Education?
}

\author{
Elkin Darío Agudelo Colorado²
}

\section{Resumen}

Este artículo de reflexión, derivado de un proceso de investigación sobre las representaciones sociales sobre Derechos Humanos en un grupo de estudiantes, indaga por el proceso de conocimiento y vivencia que tienen los estudiantes de diferentes licenciaturas de la Universidad Distrital durante su proceso formativo como docentes. En su desarrollo, se muestra cómo el campo de la representación social se origina en las vivencias cotidianas (tanto en la universidad como fuera de ella) que tienen los estudiantes en sus experiencias de conocimiento y vulneración a los DD.HH, a partir de las cuales emerge la pregunta por el sentido, el significado, la utilidad y la finalidad de los derechos en una sociedad como la colombiana y, muy particularmente, en una facultad que se dedica a la formación de licenciados en educación. Igualmente, se cuestiona la escasa voluntad política que existe para hacer más visible y concreta la Educación en Derechos Humanos (EDH) en los planes de estudio de los futuros docentes, así como en los proyectos educativos de las facultades de educación. Al final, el artículo sugiere algunas ideas fuerza para potenciar la formación de licenciados desde la perspectiva de la EDH.

\section{Palabras clave}

Formación docente, Derechos Humanos, Educación en Derechos Humanos, sujeto de derechos.

\section{Abstract}

This reflection article, derived from a research process about social representations of Human Rights in a group of students, explores the process of knowledge and experience that undergraduate students from Universidad Distrital have during their training process to become teachers. It shows how the field of social representation originates in the daily life experiences of students (both in the university and beyond) where they become aware of Human Rights violation experiences. They begin to question the meaning, significance, usefulness and purpose of rights in the contexts of societies like Colombia and particularly, in a Faculty dedicated to the teacher training. Likewise, the lack of a political will to make Human Rights Education (HRE) more visible and tangible in the curricula for future teachers is questioned, as well as the educational projects in Faculties of Education. Finally, the paper suggests some key ideas to enhance future teachers' training from a HRE perspective.

\section{Keywords}

Teacher training, Human Rights, Human Rights Education, subject of rights.

\section{Artículo recibido el 27 de octubre de 2014 - aprobado el 21 de noviembre de 2014}

1 Este trabajo se origina en los aportes ofrecidos por el seminario del Doctorado Interinstitucional en Educación UPN: El niño/a como sujeto de derechos, ofrecido por el profesor Alexander Ruiz Silva en el primer semestre de 2014 y en la investigación: Representaciones sociales sobre Derechos Humanos en un grupo de docentes en formación de las licenciaturas en Educación con Énfasis en Ciencias Sociales, Física, Artística e Inglés de la Facultad de Ciencias y Educación de la Universidad Distrital Francisco José de Caldas. El estudio se llevó a cabo en el periodo 2010-2012 con el apoyo y financiación del Centro de Investigaciones y Desarrollo Científico (CIDC) de la misma universidad y con el aval y acompañamiento del Grupo de Investigación Amautas (pedagogías críticas y formación de sujetos), al cual pertenece el autor.

2 Docente investigador de la Facultad de Educación de la Universidad Distrital, adscrito al Proyecto Curricular de Ciencias Sociales. Correo electrónico: eagudeloc@udistrital.edu.co 


\section{Introducción}

Poder conocer y entender el lugar que ocupan los DD.HH en el proceso de formación de docentes es interrogarnos por lo que viene aconteciendo en la formación de los futuros docentes como sujetos de derechos y cómo van a encarar ellos esta tarea en el desempeño de sus prácticas pedagógicas. Para dar cuenta de ello, retomo algunos testimonios de estudiantes de diferentes licenciaturas de la Universidad Distrital, a partir de los cuales se constata que el conocimiento de los DD.HH proviene más de las experiencias personales, familiares y sociales asociadas a la vulneración de sus derechos que a situaciones de disfrute y garantía de estos.

Al respecto, Magendzo (2005, pp. 31-32) nos recuerda que:

[...] Educar en Derechos Humanos en América Latina significa educar para el ejercicio de los derechos [...], esto implica partir de la visión de los oprimidos, pero que fueron "alfabetizados en derechos humanos" por haber vivido en carne propia la violación de sus derechos y haber tomado conciencia de esta práctica educativa para luchar contra la impunidad y por un estado de derecho.

Esta realidad se constata en las experiencias narradas por quienes se preparan para el desempeño de la profesión docente y que han vivido situaciones constantes de vulneración en el ámbito personal, familiar, comunitario y hasta en la propia universidad.

Sumado a esto, no se cuenta con una decisión política y pedagógica (por lo menos en los programas estudiados) que enmarque la Educación en Derechos Humanos (EDH) como un componente esencial en la formación de nuevos licenciados que, en su desarrollo profesional, se verán enfrentados a situaciones conflictivas y violentas, así como a ejercicios constantes de poder que comprometen la garantía y el disfrute de los DD.HH en las instituciones escolares, frente a lo cual los docentes tienen importantes responsabilidades éticas y políticas que cumplir por su función como pedagogos y como ciudadanos. Si bien los contenidos y pro- blemas asociados a los DD.HH en los programas de formación docente son una constante, debido al carácter público y conflictivo de los derechos y al impacto que tienen en las universidades, esto no es suficiente para llevar a cabo procesos sistemáticos de EDH que garanticen los saberes (conceptuales, jurídicos, ético-políticos) que requiere un maestro para contribuir al fortalecimiento de sus estudiantes como sujetos de derecho. Es por eso que este artículo propone al final unas pistas pedagógicas y didácticas que pueden ser consideradas para visibilizar y empoderar la EDH como parte del proceso de formación de licenciados.

\section{Referentes conceptuales ${ }^{3}$}

Este artículo no pretende establecer un debate teórico sobre el concepto formación docente, que supone además un debate histórico sobre lo que ha sido y lo que debe ser tal formación; tampoco se propone realizar un balance de la amplia producción teórica e investigativa en Colombia. Simplemente pretendo presentar algunas líneas de reflexión que aportan a esclarecer la intención de este trabajo.

La historia de la formación docente en Colombia nos devela una serie de retos que se deben emprender para transformar el imaginario social que existe sobre los maestros, así como mejorar el desempeño de las instituciones que se dedican a la formación profesional de los docentes. En ese sentido, Giroux (2003) nos recuerda que:

Uno de los puntos de partida sería reconocer la importancia de educar a los estudiantes en los lenguajes de la crítica y la posibilidad; es decir, proporcionarles a los maestros la terminología crítica y el aparato conceptual que les permitiera no solo analizar críticamente las deficiencias democráticas y políticas de las escuelas, sino también desarrollar los conocimientos y habilidades que habrán de fomentar las posibilidades para la generación

3 Las reflexiones conceptuales que presento a continuación hacen parte mi proyecto de tesis doctoral, que lleva por nombre "Educación en Derechos Humanos y constitución del sujeto de derechos. Un estudio de caso desde los programas de licenciatura de ciencias sociales de las universidades Pedagógica Nacional y Distrital de Bogotá". 
de planes de estudio, de prácticas sociales del aula $y$ de disposiciones organizativas basadas en un profundo respeto - $y$ en el cultivo de este- hacia una comunidad democrática y con fundamentos éticos [...] Dewey expresaba acertadamente la necesidad de que los educadores tomaran las consideraciones políticas y morales como el aspecto central de su educación y de su trabajo, cuando distinguía entre la "educación como una función de la sociedad" y "la sociedad como una función de la educación”. [...] Lo que aquí se debate es si las escuelas [facultades] de educación deben ponerse al servicio de la sociedad existente, y reproducirla, o si deben adoptar la función más crítica de desafiar el orden social con el fin de desarrollar y fomentar sus imperativos democráticos (p. 279).

Formar un intelectual de la pedagogía hoy no puede llevarnos a creer que el problema se resuelve única y exclusivamente abordando los dos campos de tensión, el de la formación pedagógica y el de la formación disciplinar, pues la formación ética y política del futuro licenciado es una dimensión esencial para el desarrollo de su profesión, por cuanto el docente como sujeto político y de derechos está llamado a defender un tipo de educación que garantice las libertades políticas y promueva los Derechos Humanos fundamentales. En ese sentido, es importante recordar que las instituciones que se dediquen a la formación de los docentes deben:

[...] Tener como finalidades la elaboración y experimentación de elementos que apunten hacia la construcción de un proyecto educativo y pedagógico, en estrecha relación con un proyecto político y cultural propio de las sociedades contemporáneas, en el que se afirme la pluralidad cultural, la tolerancia ideológica, el respeto a la diferencia y la lucha contra la desigualdad económica y la discriminación social y cultural (Herrera, 2001, p. 72).

En consonancia con los planteamientos anteriores, se debe anotar que la construcción de ese proyecto político y cultural para la formación de docentes necesariamente implica un proceso de deconstrucción y reconstrucción de los sentidos y las prácticas que han acompañado en nuestro país la formación de licenciados, en tanto los rasgos históricos del proceso formativo de maestros que se han esbozado a lo largo de esta aproximación nos indican la manera en que los docentes han sido funcionales a la racionalidad educativa instrumental que coloca el énfasis de los procesos educativos en las necesidades del mercado y muy poco en las necesidades del sujeto. Desde esa perspectiva, la propuesta de Giroux (1997) para repensar los procesos de formación docente resulta bastante retadora y esclarecedora, toda vez que nos permite complementar la idea esbozada por Herrera en la cita anterior e indagar en las prácticas formativas de los licenciados de ciencias sociales qué tanto nos acercamos a una propuesta de formación docente vinculada a las posibilidades de una teoría social radical que nos permita llevar a cabo ese proceso de deconstrucción pedagógica, epistemológica, ética y política.

El proyecto de "hacer" un currículum de política cultural como parte de un programa de educación del profesor consiste en asociar teoría social radical y un conjunto de prácticas estipuladas a través de las cuales quienes estudian para profesores se capacitan para desmantelar y cuestionar los discursos educativos dominantes, muchos de los cuales son víctimas de una racionalidad hegemónica e instrumental que o bien limita o bien ignora los imperativos de una democracia crítica. [...] Nosotros deseamos refundir la educación del profesor y hacer de ella un proyecto político -mejor, una política cultural- que defina a quienes estudian para profesores como intelectuales con la voluntad de establecer esferas públicas donde los estudiantes puedan debatir, asimilar y aprender el concomiendo y las habilidades necesarias para hacer realidad la libertad individual y la justicia social (Giroux, 1997, p. 219).

Pensar la formación del sujeto de derechos en las facultades de educación implica dar cuenta de qué estamos entendiendo por DD.HH y por EDH, de manera que se pueda tener elementos conceptuales para afrontar la pregunta general de este artículo: ¿quién responde por la formación de los docentes como sujetos de derechos en las facultades de educación? 
En cuanto a la definición del concepto de DD.HH, no queremos acogernos a una conceptualización cerrada (universalista) que desconozca otras formas de entender su construcción histórica, política y ética. Preferimos acogernos a un planteamiento que reconozca, como dice Boaventura Santos (1998a; 1998b), lo incompleto que se encuentra el discurso occidental de los derechos y reconocer otros aportes que enriquecen y problematizan lo construido oficialmente en la concepción de Naciones Unidas. Considerando así el asunto, me acojo a la perspectiva de Joaquín Herrera (2005) porque nos ayuda a ampliar y enriquecer la visión estrecha que hemos tenido hasta el momento respecto a la comprensión de los DD.HH:

Una teoría y una práctica de los Derechos Humanos, entendidos como productos culturales, tenderá siempre a crear, a constituir, a buscar y a constituir, a buscar y a suscitar acontecimientos, encuentros, espacios significativos de interacción que aumenten la intensidad de nuestras actitudes y aptitudes, a empoderarnos y empoderar a los otros. Para ello, debemos ampliar nuestras formas de comprensión de los Derechos Humanos y considerarlos como procesos -normativos, sociales, políticos, económicos- que abran o consoliden espacios de lucha por la dignidad humana, en otros términos: conjuntos de prácticas que potencien la creación de dispositivos y de mecanismos que permitan a todas y a todos poder hacer sus propias historias. [...] Comencemos por definir los Derechos Humanos por lo que son: productos culturales que faciliten la construcción de las actitudes y aptitudes que nos permitan poder hacer nuestras vidas con el máximo de dignidad (Herrera, 2005, p. 30).

De esta reflexión se deriva un importante imperativo ético para las facultades de educación y para el futuro desempeño profesional de los docentes en formación, por cuanto todo proyecto educativo debe construir, decidida y abiertamente, un conjunto de actitudes y aptitudes que nos permita vivir cada vez más en dignidad. Para lograr esto, las facultades de educación requieren precisar y definir propuestas pedagógicas más explícitas que hagan posible la EDH.
El trabajo de Sacavino (2012) nos muestra cómo la EDH emerge y cobra fuerza en ambientes autoritarios y cómo la acción educativa se convierte en un factor determinante para la recuperación de la democracia y la restitución de los estados de derecho. En ese sentido, no hay duda de que la educación es uno de los instrumentos más importantes para promover la propuesta de los DD.HH, por cuanto la educación es un proceso dinámico, complejo y social que conecta los sentires y necesidades de los seres humanos con los valores culturales y políticos que ha creado la humanidad para alcanzar mayores niveles de felicidad y convivencia pacífica. Una de las diversas y ricas definiciones sobre $\mathrm{EDH}^{4}$ que satisface, en parte, nuestra apuesta es la siguiente:

Significa que todas las personas -independientemente de su sexo, origen nacional o étnico y sus condiciones económicas, sociales o culturalestienen la posibilidad real de recibir educación sistemática, amplia y de buena calidad que les permita: comprender sus Derechos Humanos y sus respectivas responsabilidades; respetar y proteger los Derechos Humanos de otras personas; entender la interrelación entre Derechos Humanos, estado de derecho y gobierno democrático; y ejercitar en su interacción diaria valores, actitudes y conductas consecuentes con los Derechos Humanos y los principios democráticos. Entendemos este derecho a la educación en Derechos Humanos como parte del derecho a la educación y como condición necesaria para el ejercicio efectivo de todos los Derechos Humanos.

[...] Es común que la Educación en Derechos Humanos sea circunscrita muchas veces a "capacitación en Derechos Humanos", que es apenas una faceta de aquella. El Instituto Interamericano

4 El pedagogo chileno Abraham Magendzo sostiene: "se ha definido el término de educación en Derechos Humanos como la práctica educativa que se funda en el reconocimiento, la defensa, el respeto y la promoción de los Derechos Humanos y que tiene por objeto desarrollar en los individuos y en los pueblos sus máximas capacidades como sujetos de derechos y brindarles las herramientas y elementos para hacerlos efectivos. Se trata de una formación que reconoce las dimensiones históricas, políticas y sociales de la educación y que se basa en los valores, principios, mecanismos e instituciones relativos a los Derechos Humanos en su integralidad y en su relación de interdependencia e indivisibilidad con la democracia, el desarrollo y la paz" (2005, p. 31). 
de Derechos Humanos (IIDH) considera que la educación en derechos humanos es más bien un eje transversal del derecho a la educación que debería estar presente en lo sustantivo y metodológico y en todas las formas posibles de enseñanza formal e informal. Incluso debe estar presente en procesos culturales y en toda dinámica que incida en el reforzamiento y reformulación de patrones culturales (Instituto Interamericano de Derechos Humanos, 2003, p. 12).

Finalmente, toda esta riqueza de argumentos, reflexiones y definiciones nos lleva a dar cuenta de la categoría sujetos de derechos como la expresión conceptual que le da sentido a la pregunta central y transversal de este artículo. Al igual que las anteriores categorías, la de sujeto de derechos entraña una diversidad de posturas y énfasis que enriquecen su comprensión y problematización. Para el filósofo francés Paul Ricœur, la capacidad es un aspecto central al momento de preguntarnos: ¿quién es el sujeto de derechos? En ese sentido, nos recuerda que al sujeto de derechos "le faltan las condiciones de actualización de sus aptitudes. Estas tienen necesidad de una mediación continua de formas interpersonales de alteridad y de formas institucionales de asociación para poder llegar a ser poderes reales a los que corresponderían derechos reales" (1999, p. 43).

Habría que decir también que el sujeto de derechos es un sujeto de poder por cuanto se asume como una persona digna de respeto consigo mismo y con los demás, implica desplegar todas sus capacidades para exigir el disfrute de sus derechos cuando estos están siendo pisoteados tanto a él como a un semejante. Desde esta postura, considero que la EDH tiene un papel muy importante por cumplir en las facultades de educación, pues es necesario romper las lógicas de poder que han hecho de las instituciones escolares espacios para la negación y el silencio, en las que los sujetos tienen profundas limitaciones para desplegar sus capacidades y hacer valer sus derechos. Por eso vale la pena tener en cuenta las precisiones que Sacavino toma de Candau:

Ser sujeto de derechos implica reforzar en la vida cotidiana, a través de prácticas concretas, la lógica expansiva de la democracia, afirmar el principio y el derecho de la igualdad, establecidos en la esfera jurídica y política y hacer extensiva esa dinámica igualitaria a las diversas esferas de la sociedad. Formar la conciencia de ser sujeto de derechos significa también poder desarrollar, en la práctica y en la construcción de la ciudadanía, la articulación de los derechos de igualdad con los de la diferencia, así como los derechos individuales con los derechos colectivos (Sacavino, 2012, p. 92).

Una vez establecidas las referencias conceptuales en las que se enmarca este artículo de reflexión, pasemos ahora a presentar cómo es que los estudiantes que se forman para ser docentes conocen los DD.HH en el contexto de las facultades de educación y qué tanto esto redunda en su formación como sujetos de derechos.

\section{¿Cómo conocen los DD.HH los docentes en formación en las facultades de educación? ${ }^{5}$}

La lucha de los profesores en defensa de sus derechos y de su dignidad debe ser entendida como un momento importante de su práctica docente, en cuanto práctica ética. No es algo externo a la actividad docente, sino algo intrínseco a ella. El combate a favor de la dignidad de la práctica docente es tan parte de ella misma como el respeto que el profesor debe tener a la identidad del educando, a su persona, a su derecho de ser (Freire, 2010, p. 64).

Esta exigencia implica que la Educación en Derechos Humanos (EDH) en las instituciones formadoras de docentes deje de ser un pedido externo que demandan distintos instrumentos políticos y jurí-

5 Los testimonios que sustentan este apartado hacen parte de la investigación: Representaciones sociales sobre Derechos Humanos en un grupo de docentes en formación de las licenciaturas en Educación con Énfasis en Ciencias Sociales, Física, Artística e Inglés de la Facultad de Ciencias y Educación de la Universidad Distrital FJC. Esta investigación, de corte cualitativo, adoptó la investigación-acción (IA) como método que orientó el proceso investigativo. Latorre -citando a Elliot- define la investigación-acción como "un estudio de una situación social con el fin de mejorar la calidad de la acción dentro de la misma" (2007, p. 24). Basados en este método de investigación, se seleccionaron cuatro técnicas de recolección de información: entrevista en profundidad, relatos de vida, grupos de discusión y la asociación libre de palabras, técnica propia de la psicología social. 
dicos en los ámbitos nacional e internacional, y se asuma como una intención cognitiva, ética, política y jurídica que permea los programas curriculares de formación docente, toda vez que del desempeño profesional de los pedagogos se espera una actitud y una aptitud de la más alta calidad humana, no solo para respetar la integridad y dignidad de sus estudiantes, sino, ante todo, para facilitar las herramientas que le permitan al estudiante -niño, niña, joven- asumirse y proyectarse como un sujeto de derechos.

Es decir, "se trata de poner a cada sujeto en situación de discernir y tomar posición, con información suficiente y en la búsqueda de criterios argumentativos fundados" (Siede, 2007, p. 155), para lo cual los docentes, a través de sus prácticas pedagógicas, deben procurar reemplazar la acumulación de información por un trabajo tendiente a ayudar a los estudiantes a comprender y conceptualizar la realidad, por cuanto los DD.HH son una importante herramienta que involucra aspectos relacionados con la historia, los conceptos, argumentos éticos y políticos, así como los mecanismos de acción y protección tendientes a prevenir o restituir los derechos vulnerados.

Sin embargo, al analizar lo que acontece en los programas de formación de docentes, encontramos que dicha exigencia y finalidad se da de manera fragmentada y temerosa, o por lo menos eso es lo que reflejan los planes de estudios con los que se forman los maestros en las facultades de educación, especialmente de las universidades estatales. Tal parece que el contenido y la vivencia de los DD.HH llegan a los estudiantes por su condición de vulnerabilidad, por los conflictos que enfrentan a diario en los barrios y localidades, en las mismas universidades, por las secuelas del conflicto armado, entre otras razones. Al respecto, al preguntarle por situaciones o experiencias vividas en los últimos tiempos, relacionadas con la vulneración a sus derechos, un estudiante de la Licenciatura de Física afirma:

[...] En este año sí me ha pasado muchas veces, me pasó que íbamos con unos compañeros por la [carrera] séptima y había un señor que tenía una publicidad contra Uribe ${ }^{6}$ que decía: "no a las armas gringas, no más Uribe tres ${ }^{7}$, no al TLC” y entonces había unos policías que le decían que se tenía que retirar del "septimazo" porque no era permitido hacer esa publicidad; entonces entramos nosotros con los compañeros a alegar pero ya entramos con un poco más de argumentos políticos, decir que se está violando el derecho a la libre expresión, que el señor no estaba agrediendo a nadie para que ellos utilizaran la fuerza. Como te decía, nosotros tenemos un medio de comunicación alternativo y ahí publicamos fotos, y pues claro, nosotros íbamos con nuestras cámaras y de inmediato empezamos a sacar fotos a los policías, entonces ellos como que ya se abstuvieron un poco, en ese sentido sirvió estar metido en todo este rollo como tal de las comunicaciones, de los Derechos Humanos, pero lo que te decía, o sea, el entrar así como tal a defender los derechos alivia pero no quita el problema, porque el problema es estructural y es mucho más complejo (relato de vida, Licenciatura en Física).

Como puede observarse, el estudiante reconoce que sus argumentos éticos y políticos para defender los DD.HH provienen de su experiencia en un colectivo de comunicaciones que le ha permitido conocer la problemática de los DD.HH que enfrenta el país. Nótese que su testimonio no da cuenta de los aportes académicos que le brinda la facultad de educación, sino que estos provienen de la organización social a la que pertenece. Sobre esto valdría la pena preguntarnos: ¿cuántos estudiantes que se preparan para ser profesores tienen el interés y la posibilidad de participar en escenarios sociales que los lleven a conocer el contenido y la propuesta de los DD.HH? ¿Por qué a las directivas de una licenciatura en Física no se les puede ocurrir que, además del saber disciplinar y el saber pedagógico, es necesario incorporar otros

6 Se refiere al expresidente de Colombia Álvaro Uribe Vélez, elegido para el periodo 2002-2006 y reelegido para el siguiente periodo (2006-2010) gracias a una reforma constitucional impulsada por su gobierno.

7 La expresión Uribe 3 hace referencia a un posible tercer periodo presidencial de Uribe. En 2010, la Corte Constitucional declaró inexequible la ley convocatoria de referendo mediante el cual se pretendía forzar una nueva reforma constitucional que permitiera una segunda reelección inmediata. 
saberes transversales que requiere el futuro docente para su desempeño profesional? ¿Acaso la dignidad $\mathrm{y}$ el respeto no son asuntos que le competen a los profesores de Física en el marco de sus relaciones con niños, niñas y jóvenes en la escuela?

Bien vale la pena aquí citar las palabras de Candau et al. cuando nos recuerdan que:

Si una de las finalidades de la escolarización es la formación para la ciudadanía, lo que supone colaborar en la construcción de sujetos de derechos, la Educación en Derechos Humanos está llamada a constituirse en un eje fundamental de la escolarización. Para tal, la formación de los profesores en esta perspectiva se configura como una necesidad y una urgencia $(2013, \text { p. } 59)^{8}$.

Sin embargo, dicha necesidad y dicho imperativo para los programas de formación docente choca con una serie de barreras que impiden su reconocimiento y visibilización en los planes de estudios de las diferentes carreras, pues lamentablemente la formación de licenciados se ve limitada, ya que quienes las dirigen privilegian la formación disciplinar en detrimento del saber pedagógico y, más aún, del saber orientado a la formación de los profesores como sujetos de derechos. Sobre esto, dos estudiantes de licenciatura en Inglés y en Física comparten esta visión:

[...] Los profesores no le ven tanta importancia a los Derechos Humanos, aunque es un aspecto vital. Yo pienso que se enfocan más en la parte de formación académica, es decir, en enseñar los contenidos disciplinares y la parte digamos pedagógica, pero no incluyen los Derechos Humanos como eje de formación. La verdad yo no sé cuál es la razón por la cual los profesores dejan a un lado los DD.HH, por qué no los tienen en cuenta (entrevista, Licenciatura en Inglés).

No conozco ninguna asignatura o cátedra sobre DD.HH dentro del proyecto. Es que todavía creo que manejamos un currículo muy cerrado, la mayoría le da importancia a las asignaturas que tiene que ver con la disciplina (la Física), pues toda

8 Traducción libre del portugués. la carga académica se va hacia la disciplina; es muy poco lo que se orienta respecto a la pedagogía, por ejemplo, si tú ves, la carga académica en pedagogía no es que sea muy fuerte, mucho menos existe un espacio o una cátedra para DD.HH (entrevista, Licenciatura en Física).

Tal y como se observa en los anteriores testimonios, los estudiantes de la facultad perciben desinterés en los profesores para incluir y desarrollar contenidos relacionados con los DD.HH y la EDH en los planes de estudio de sus respectivas licenciaturas, en el sentido de que ni siquiera alcanzan a considerarlo como un aspecto relevante para la formación de licenciados y su futuro desempeño profesional.

Si bien en esta reflexión hace referencia a experiencias específicas que se dan en el marco de una facultad de educación y que nos indican la ausencia de un eje de formación específico en DD.HH, llama poderosamente la atención que el Plan Nacional de Educación en Derechos Humanos (PLANEDH), al aproximarse a un diagnóstico de la EDH en el país, concretamente sobre docentes y agentes educativos, afirme los siguiente:

Se entiende que son personas que se reconocen como sujetos de derechos con formación y sentido de su acción educadora, es decir, con una intencionalidad política definida, que hacen acopio de un saber en Derechos Humanos, con competencia pedagógica y metodológica para generar la movilización de pensamiento y/o la acción en Derechos Humanos.

En relación con el grado de inclusión de los Derechos Humanos como contenido de la formación de los agentes educativos, las hipótesis que asume el PLANEDH es la siguiente:

El país no cuenta con un sistema estructurado de formación de agentes educativos que integre de manera sistemática la reflexión pedagógica y la didáctica sobre la EDH.

La existencia de cátedras y asignaturas, de cursos y talleres y aún de algunos postgrados no dan cuenta per se de un sistema estructurado de agentes educativos. Para que ello suceda es necesario: 
- Incidir en los programas de estudio para incorporar la formación en pedagogía y metodologías didácticas correspondientes a la formación de los profesores antes y después de su entrada en funciones $^{9}$ (PLANEDH, 2009, pp. 59-60).

Contrario a lo que sucede en la educación formal, en la educación popular, que se gesta en las dinámicas de las ONG y los movimientos sociales, la EDH ha sido adoptada e incorporada como eje estructurante de formación, debido a que gran parte de su esencia educativa corresponde a procesos de promoción, defensa y reivindicación de DD.HH que demandan con urgencia las poblaciones a las que se dirige su acción organizativa.

Por el contrario, las instituciones escolares no demandan procesos educativos que promocionen el conocimiento y defensa de los DD.HH, pues allí su desconocimiento e irrespeto se vive a flor de piel. La escuela, como poder constituido, está controlada y determinada por relaciones de poder que se afirman con el desarrollo de políticas públicas, las cuales, de una u otra manera, limitan el desarrollo y empoderamiento de poderes constituyentes que afirmen la propuesta ético-política de los DD.HH como un discurso emancipador en el contexto escolar. Tal poder constituyente es capturado y absorbido por el poder constituido y representado; al decir de Martínez (2007, p. 105), "el poder constituyente queda encerrado en figuras políticas abstractas, que siguen produciendo un sujeto despolitizado, pasivo, que se convierte en fuente de legitimidad y no de poder" ${ }^{\prime 0}$.

9 EI PLANEDH presenta diversas estrategias para fortalecer la formación docente en este campo, aunque aquí solo se cita la que directamente tiene relación con la formación de profesores.

10 Un buen ejemplo de esto es lo que ha venido sucediendo con los consejos de estudiantes después de su reconocimiento e institucionalización legal que les confirió la Ley General de Educación y concretamente el decreto reglamentario 1860 de 1994. Estos consejos, al quedar atrapados por la fuerza constituida de la ley y la formalidad de las instituciones educativas, perdieron su carácter crítico, movilizador y beligerante y se convirtieron en una caja de resonancia de las directivas educativas con escasas posibilidades de transformación, contrario a lo que sucedía con los consejos estudiantiles cuando se gestaban al margen o en la clandestinidad de los poderes institucionales. Al respecto, uno de los apartados del artículo 29 del Decreto 1860 afirma: "el Consejo Directivo deberá convocar, en una fecha dentro de las cuatro primeras semanas del calendario académico, sendas asambleas integradas por los alumnos que cursen cada grado, con el fin de que elijan de su seno,
Si bien las propuestas del Movimiento Pedagógico de la década de los ochenta y comienzos de los noventa, incorporadas, por demás, en el marco normativo de la Ley General de Educación, tuvieron una amplia difusión y recepción en las escuelas, esas mismas iniciativas fueron recibidas e implementadas con la misma racionalidad técnico-instrumental que caracteriza el funcionamiento de estas instituciones y rápidamente perdieron su carácter crítico y transformador al caer en la lógica del disciplinamiento y el control que invisibilizan al estudiante y a un número representativo de docentes como sujetos de derechos. Esto lo ilustra claramente Mclaren cuando sostiene:

Por un lado, los neoconservadores han definido a la escuela como un apéndice del mercado laboral, un subconjunto de la economía, y encubren su análisis con la jerga tecnocrática de la teoría del capital humano. Por el otro, los liberales han criticado más a fondo la instrucción escolar, pero hasta ahora han sido incapaces de abordar los problemas más graves en el interior de las escuelas: una sociedad dividida en razas, clases y géneros. En consecuencia, los preceptos resultantes para la reforma escolar han quedado seriamente limitados a formas de reformismo burgués despojadas de una crítica concertada del capitalismo. [...] Pasivamente, han entregado la reforma educativa a una devoción ciega por el procedimiento, más que demostrar un interés por los objetivos sociales emancipatorios. Además, las pedagogías de tipo administrativo cada vez más populares han dado por resultado la proposición de políticas que promueven despojar a los maestros de sus habilidades y crear una racionalidad tecnocrática en la cual la instrumentación está desprovista de planeación y concepción, y el modelo dominante de lo que es el maestro se convierte en un técnico u oficinista de cuello blanco (2005, pp. 82-83).

mediante votación secreta, un vocero estudiantil para el año lectivo en curso". Como bien se observa, el derecho a la participación de los estudiantes en un asunto que queda a discreción de las directivas, es decir, la participación como derecho e iniciativa de cada ciudadano estudiante queda capturada por el control constituido de las normas. 
Romper con esta racionalidad instrumental y procedimental que ha convertido a los docentes en técnicos y operarios, en detrimento de su condición como intelectuales de la pedagogía, implica que las facultades de educación asuman la tarea aplazada de incorporar en los currículos que orientan la formación de docentes una perspectiva crítica de formación ético-política basada en el conocimiento, vivencia y defensa de los DD.HH, en tanto que el discurso crítico de los mismos no asume los DD.HH como pretensiones, sino como oportunidades y capacidades de realización eficaz.

Un docente formado en la perspectiva de los DD.HH como posibilidad, como realización eficaz, difícilmente perderá de vista en el desarrollo de sus prácticas pedagógicas que el estudiante es un sujeto que merece toda consideración y reconocimiento, por cuanto el escenario en el que acontece la enseñanza-aprendizaje debe ser concebido como un espacio-tiempo para la garantía y disfrute de derechos, lo cual aumentaría de manera significativa una cultura ciudadana de promoción y respeto a los DD.HH.

Ahora bien, lo que viene aconteciendo en los procesos de formación de docentes es que el conocimiento y vivencia de los DD.HH es un proceso externo a la formalidad de los planes de estudio de las diferentes licenciaturas, ya que, como se vio en los testimonios de los estudiantes unas páginas arriba, estos no han alcanzado un reconocimiento como eje estratégico de la formación de docentes; sin embargo, la crítica situación humanitaria que vive el país por efecto de múltiples violencias, entre las que sobresale la violencia estructural, la violencia directa por efecto del conflicto armado y la violencia criminal urbana, así como las medidas de control y represión de la fuerza pública posibilitan que la pregunta por el sentido y el cumplimiento de los DD.HH sea una constante en la vida de quienes se preparan para el ejercicio profesional de la docencia, pues la vulneración de los derechos es una situación que viven a lo largo de su vida cotidiana, tanto en la universidad como en sus territorios de residencia, de militancia social y de ocio. Veamos algunos testimonios al respecto:
Hace poco algo que nos causó mucha molestia entre los estudiantes fue la re-instalación de las dichosas cámaras [de vigilancia] y pues el argumento era que se hacía por seguridad, pero realmente los lugares donde las instalaron no son lugares, digamos, que tengan que estar vigilados, a no ser por los mismos estudiantes, entonces, eso se convirtió como en una violación a la intimidad, como un seguimiento ya muy permanente $y$, no sé, se siente uno como observado todo el tiempo y eso causó muchas molestias y pues hubo protestas y ya sabemos todo lo que sucedió, los estudiantes se organizaron y las hicieron quitar, entonces ha habido muchas manifestaciones y pedreas, por ejemplo, ahora lo del presupuesto, pues realmente esto es como ya muy colombiano, entonces uno ya como que aprende a convivir con esto y se le hace normal, todo esto son como agresiones que no son de una forma física ni directa, pero sí una agresión que compromete el derecho a la educación de todo el pueblo (entrevista, Licenciatura en Educación Artística).

La minga humanitaria a favor de los indígenas awa, a quienes las FARC les mataron ocho de sus integrantes, eso fue en mayo de este año, ellos hicieron minga y llevaron gente de derechos humanos, de sus comunidades, de la ONIC y pues ahí se estuvo acompañando como estudiantes dos semanas y media, pues nosotros particularmente hicimos algunos programas, se tomaron fotos, se sacaron videos y todo ese material lo ayudamos a difundir para que la gente conozca lo que están viviendo estas comunidades (Entrevista, licenciatura en Física).

[...] Yo me encontraba rumbeando con unos amigos del barrio y como se nos acabó el trago decidimos salir a comprar, pero ya las tiendas se encontraban cerradas, de un momento a otro aparecieron los señores policías, salieron de la nada, nos abordan, nos piden nuestros papeles y nos preguntan qué hacemos ahí, nosotros le comentamos la situación muy decentemente, pero uno de ellos, como dicen popularmente, se enamora de nosotros y no sirvió ninguna explicación y nos fueron subiendo de una vez a la patrulla y nos llevaron a la UPJ 24 horas, simplemente porque queríamos comprar un vino, entonces a mí me 
parece una violación de los Derechos Humanos que dice, precisamente, que nadie puede ser arbitrariamente detenido, preso ni desterrado. Nos metieron a todos, así, en grupo a esas bodegas, pero en ningún momento nos volvieron a preguntar nada, sino simplemente nos encerraron, no nos dieron tiempo de hablar con nadie ni de defendernos, nada, simplemente: ¡Adentro 24 horas! Después de salir yo decía: “¡Este país es una mierda! Aquí no se respetan los derechos de la gente, menos si uno es joven" (relato de vida, Licenciatura en Educación Artística).

La experiencia de vínculo con los DD.HH para los estudiantes de las facultades de educación, especialmente quienes se forman en las universidades estatales, es una práctica que surge de vivenciar o conocer situaciones profundas de indignidad, las cuales desatan en ellos otros sentimientos relacionados con la desesperanza, el miedo, la rabia y, en ocasiones, sentimientos de solidaridad con las víctimas, tal y como lo expresa el estudiante de la Licenciatura en Física de la Universidad Distrital que se solidariza con la situación de los indígenas awa del departamento de Nariño, no como parte de su práctica pedagógica ni como parte de los programas de extensión de su Facultad, sino como parte de los compromisos sociales que adquiere en el escenario universitario no formal-informal, el cual genera una serie de dinámicas organizativas constituyentes que se gestan por fuera de los planes de estudio de su licenciatura, es decir, en experiencias ligadas a la educación popular ${ }^{11}$.

Ahora bien, ¿cómo entender en el contexto de las facultades de educación que la propuesta éticopolítica de los DD.HH es un asunto que, además de conocimientos, implica un proceso de involucramiento en las realidades sociales en las se ponen en juego su vigencia y garantía, que implica apropiarse de los mecanismos para su protección, generar acciones que reconozcan y visibilicen al otro como sujeto de derechos y ser cuidadoso del respeto y la dignidad de los demás?

11 Al respecto, Abraham Magendzo (2005, pp. 19-20) nos recuerda: “Es importante hacer ver que la educación no formal ha estado históricamente ligada a la defensa de los derechos humanos, de suerte que se ha entendido que educación sin defensa no tiene sentido, así como la defensa es un proceso educativo".
Comprender este imperativo implica que esta responsabilidad no se deje única y exclusivamente a las dinámicas educativas en las que se involucran los estudiantes por fuera de la formalidad de los planes de estudio. Se requiere que las facultades de educación incluyan los DD.HH en el proceso formativo no como algo marginal, sino como un dispositivo que afecte los diferentes escenarios académicos articulados al Proyecto Educativo de Facultad, a los planes de estudio de las diferentes licenciaturas, a los grupos y semilleros de investigación, a los programas y proyectos de extensión, entre otros espacios que inciden en la formación de los fututos docentes.

Es importante considerar que la EDH es parte integral del derecho a la educación, tal y como lo sostienen el Instituto Interamericano de Derechos Humanos. Por tanto, debe ser reconocida en los programas de formación de docentes, con lo que se busca que el desarrollo de las prácticas pedagógicas que llevan a cabo los docentes doten a los niños, niñas y jóvenes de los conocimientos, las habilidades y las actitudes necesarias para el ejercicio y la defensa de sus derechos.

De aquí se desprende que los docentes tienen una gran responsabilidad en la materialización del derecho a la educación y dentro de esta, el derecho a la EDH como parte de las posibilidades que potencian a niños, niñas y jóvenes para el ejercicio de una ciudadanía crítica y responsable; sin embargo, no se puede entender la falta de un programa estructurado que forme a los docentes en el conocimiento, promoción y defensa de los DD.HH como complemento del saber disciplinar y pedagógico, por cuanto el docente como tutor y acompañante del proceso de enseñanza-aprendizaje debería, en principio, contar con las herramientas necesarias para que sus estudiantes aprendan a conocer y defender sus derechos, así como a promover y respetar los de los demás, responsabilidad que atañe no solo a los licenciados de ciencias sociales, sino al conjunto de los docentes que se forman para el ejercicio de su profesión.

Las facultades de educación tendrían que asumir el reto de formar nuevos docentes con la suficiente inteligencia, creatividad y voluntad política para 
romper con la lógica de la escuela convencional, que, está claro, es incapaz de preguntarse por los DD.HH. Sobre esto, el profesor Álvarez (2010, p. 152) enfatiza que:

La escuela es el símbolo del disciplinamiento y el adoctrinamiento y, en general, una institución que viola los derechos de los niños y las niñas. Por eso no se puede entender una política pública en educación que amplíe la cobertura, que escolarice a la población, sin cuestionar lo que pasa por dentro de la escuela. No se trataría simplemente de crear más escuela; la educación entendida como un derecho humano no es solo más escuela para todos, sino qué tipo de escuela; implica, incluso, por momentos, luchar contra lo que la escuela hace, en términos de violencia, de discriminación, de segregación, de dominación.

Ahora bien, una política pública que rompa este cerco requiere de un cuerpo de docentes que se formen con la concepción de asumir y entender a los niños, las niñas y los jóvenes como sujetos de derechos, es decir, como sujetos que, en el marco de la exigibilidad del derecho a la $\mathrm{EDH}$, requieren docentes con capacidad de responder a esa exigencia ética y política que demandan las actuales generaciones.

\section{Algunas pistas pedagógicas y didácticas para promover, en el contexto de las facultades de educación, procesos de formación en DD.HH con los estudiantes que se preparan para el ejercicio de la docencia ${ }^{12}$}

Tal y como se anunció al comienzo de este artículo, el estudio realizado en diferentes programas de licenciatura de la Facultad de Educación de la Universidad Distrital acerca de la representación social sobre de los DD.HH, arrojó que el núcleo central de las representaciones se define a partir de las experiencias y vivencias de orden personal, familiar, social y universitario que conectan al estudiante con la realidad y los problemas que obstaculizan el disfrute y la garantía de los DD.HH.

12 Para profundizar en este aspecto, recomiendo consultar Agudelo, 2012.
De este modo, los docentes en formación conocen algo del contenido, de la jurisprudencia y de los mecanismos de protección de derechos como consecuencia de las vulneraciones que han vivido en el plano personal, familiar, social o universitario y no como consecuencia del disfrute y el respeto de los mismos en sus vidas. Así, la pregunta por la dignidad es respondida a partir de experiencias de indignidad que los han marcado y los han llevado, por necesidad de protección, a acercarse al discurso y la propuesta de los DD.HH.

Esto, a su vez, nos llevó a constar que el estudiante de la facultad de educación, al momento de enfrentarse a una situación de vulneración de sus derechos o de los de personas cercanas, no cuenta con los conocimientos necesarios para enfrentar dichas situaciones en las que se vio comprometida su dignidad, es decir, ni su paso por la educación básica y media ni por la educación superior lo han capacitado y formado para el ejercicio de sus derechos a partir del conocimiento y uso de los cuerpos políticos y normativos que existen para tal finalidad.

No se entiende cómo un país como Colombia, que tiene uno de los índices más altos en violación a los DD.HH y un alto porcentaje de impunidad en su sistema judicial, no lleve a cabo un trabajo profundo de EDH con unos de los actores principales del sistema educativo, los maestros.

Al respecto, quisiera poner a consideración algunas ideas-fuerza que contribuyan a fortalecer la formación de docentes en las facultades de educación, con el fin de potenciar el reconocimiento y el empoderamiento de las niñas, los niños y jóvenes como sujetos de derechos:

- En primer lugar, es necesario que en los programas de formación de docentes, más específicamente en los planes de estudio, se habiliten espacios de conocimiento explícito de los DD.HH como un área del saber pedagógico, político y social, en dirección a que se respeten los derechos de los estudiantes, se identifiquen los derechos efectiva o potencialmente vulnerados y se desarrollen las habilidades necesarias para la utilización de los instrumentos 
y mecanismos de defensa y protección. Esto requiere, entre otras medidas, tomar decisiones políticas que permitan la visibilización e inclusión de los DD.HH en los proyectos educativos de facultad, en los propósitos formativos de las diferentes licenciaturas, en la identificación de núcleos temáticos y problemáticos relacionados con los DD.HH y su educación, de tal manera que puedan ser abordados a través de diferentes campos de formación (disciplinar, pedagógico, científico, ético-político y estético-comunicativo); igualmente, potenciar la creación de grupos y semilleros de investigación en el campo, así como de programas de extensión que les permita a los estudiantes situar e intervenir contextos educativos formales e informales. De lo que se trata, en suma, al potenciar la enseñanza de los DD.HH en los programas de formación de docentes es de:

- Reconocer que esos derechos no son un adorno de estilo humanístico para unos pocos, sino que de suyo revalorizan a los sujetos en el preciso sentido de configurarles como sujetos más educados, más agentes de sí mismos, generadores de cambios deseables para sí y para su entorno social y material (Gil y Jover, 2008, p. 243).

- En segundo lugar, los estudiantes de diferentes licenciaturas coinciden en afirmar, a través de sus testimonios, que la EDH no es un asunto que se agote única y exclusivamente en conocimientos históricos, políticos y jurídicos; además de esto se requiere abordajes que involucren el afecto y la sensibilidad frente al cuidado del otro, es decir, como alguien que merece igual respeto y dignidad. Cuando se les preguntó a estos maestros en formación: ¿de qué manera incorporarían el conocimiento y práctica de los DD.HH en la enseñanza de las áreas básicas y fundamentales cuando se desempeñen como docentes en las instituciones escolares?, un estudiante respondió:

Pues me niego totalmente a que mis chicos y chicas se aprendan de memoria los Derechos
Humanos con toda su doctrina y todo lo que hay de ellos en las constituciones y decretos, a eso yo me niego, me parece que eso no sirve de mucho. Hay que enseñar a sentirlos y hay que enseñarles a negociar con la vida misma y con el otro, o sea, negociar en el buen sentido es dialogar, decirle "usted qué piensa, qué podemos hacer frente a tal cosa", y que el otro comparta también sus posturas y podamos hacer un trabajo conjunto. Por eso lo que vaya a hacer en mi colegio a través de mis cátedras y planes de estudio es trabajar los derechos humanos de una manera pedagógica y didáctica radicalmente distinta, pues no se trata de enseñar por enseñar, no se trata de imponer un conocimiento, de lo que se trata es que los derechos humanos nos sirvan para vivir mejor y para eso tenemos que enseñar a sentirlos (entrevista, Licenciatura en Ciencias sociales).

Si bien es fundamental en el proceso de formación de licenciados el conocimiento de los cuerpos normativos con sus historias, sus enfoques e instrumentos, también, como destaca este docente en formación, es necesario aprender a sentir los DD.HH, pues un profesor que no se conmueva y se solidarice con la suerte de aquellos a quienes han ultrajado su dignidad, difícilmente podrá comprometerse con la defensa de derechos en abstracto. En ese sentido, el profesor Hoyos nos plantea una interesante idea que bien podría tenerse en cuenta para el proceso de formación de licenciados, cuando afirma:

Como es bien sabido, Strawson (1974) elige tres sentimientos de especial significación con respecto a la conciencia moral: el resentimiento devela una interrelación originaria en la que nos encontramos en el mundo de la vida, la cual es violada por aquel con quien nos resentimos porque consideramos que él es consciente de haber querido romper ese vínculo humano. El sentimiento de indignación es compartido por nosotros cuando nos damos cuenta de que un tercero injuria a otro como si lo hubiera hecho con usted o conmigo. Y en el sentimiento de culpa nos avergonzamos de la ofensa provocada a otro (Hoyos, 2012, p. 6).

Por tanto, una cultura de los DD.HH en el contexto de las instituciones educativas, que se oriente al reconocimiento de las niñas, los niños y los jóve- 
nes como sujetos de derechos, se construye a partir del fortalecimiento de la sensibilidad moral de las personas, la misma que nos permite indignarnos cuando se desconocen y se vulneran los derechos de los estudiantes y nos conduce a emprender acciones a favor de su restitución y respeto.

- Finalmente, vale la pena resaltar que las vivencias y experiencias de los maestros en formación que enlazan los DD.HH ponen de presente la importancia de las organizaciones sociales y comunitarias y de las organizaciones estudiantiles, como fuente invaluable de saberes que les han permitido comprender el sentido e importancia de los derechos para el ejercicio de una ciudadanía activa y crítica, así como para el ejercicio profesional de la docencia.

Teniendo esto en cuenta, es clave para las facultades de educación que los programas de formación de maestros orienten una parte de su trabajo hacia la relación estrecha con este tipo de instancias productoras de sentidos y de saberes alrededor de los DD.HH, toda vez que, como afirma Torres (2004, p. 64):

La producción más rica de conocimiento y pensamiento social no se está generando desde el centro de las instituciones que el proyecto moderno creó para la producción del conocimiento, las ciencias sociales clásicas; sino más bien desde sus márgenes, en otros campos del saber no disciplinar o, incluso, por fuera de las instituciones universitarias.

Esta relación no puede limitarse a las iniciativas individuales de los formadores; debe construirse una política de diálogo y de encuentro con estas experiencias, de tal manera que el conocimiento que se produce en y desde ellas pueda dialogar con los saberes producidos en las facultades de educación. De esa manera, el conocimiento sobre los DD.HH ha de construirse con base en ejercicios concretos de promoción, denuncia y reivindicación y no solo como un conocimiento aislado de la realidad social y política que viven los futuros docentes y el país.

Esta relación con las organizaciones sociales arroja como saldo pedagógico-político que la mejor $\mathrm{EDH}$ que se puede llevar a cabo con los educadores es el aprendizaje de la propia defensa de los DD.HH. De esa manera, reivindicar a niños, niñas y jóvenes en su condición de sujetos de derechos será una constante a lo largo de las prácticas pedagógicas, es decir, un estilo de vida que acompaña el quehacer educativo y no un tarea más que se impone externamente a la ya extensa lista de tareas que enfrentan a diario los docentes en las instituciones escolares.

Esto nos lleva a considerar la necesidad de que las facultades de educación vean en las organizaciones-movimientos sociales un sujeto educativo, tal y como lo propone Zibechi:

Que el movimiento social se convierta en un sujeto educativo y que, por tanto, todos sus espacios, acciones y reflexiones tengan una "intencionalidad pedagógica”, me parece un cambio revolucionario respecto a cómo entender la educación, y también a la forma de entender el movimiento social (Zibechi, citado en Nuin, 2008, p. 163).

Valorar y visibilizar las pedagogías en movimiento que caracterizan a los movimientos sociales es posibilitar que la experiencia y el saber de sus organizaciones sea reconocido como fuente de conocimiento $y$ de acción ético-política en los planes de estudio que orientan la formación de los docentes.

\section{Referencias bibliográficas}

Agudelo, E. (2012). Entre pupitres y derechos: conocimientos, vivencias y representaciones de los derechos humanos en los estudiantes de educación de la Universidad Distrital. Informe final de investigación. Bogotá: Universidad Distrital-Centro de Investigación y Desarrollo Científico.

Álvarez, A. (2010). Claves para leer la educación como un derecho humano. En Restrepo. M., Sarmiento, A., Fariñas, M. et al., Teoría crítica de los Derechos Humanos. Tunja: UPTC.

Candau et al. (2013). Educação em direitos humanos e formação de professores(as). Sao Paulo: Cortez Editora.

Freire, P. (2010). Pedagogía de la autonomía. Saberes necesarios para la práctica educativa. Buenos Aires: Siglo XXI.

Gil, F. y Jover, G. (2008). La educación en la ética de los derechos humanos. En Hoyos, G. (ed.), Filosofía de la 
educación. Enciclopedia Iberoamericana de Filosofía (pp. 229-250). Madrid: Trotta.

Giroux, H. (1997). Los profesores como intelectuales. Hacia una pedagogía crítica del aprendizaje. Barcelona: Paidós.

Giroux, H. (2003). La escuela y la lucha por la ciudadanía. México: Siglo XXI.

Herrera, J. (2005). Los derechos humanos como productos culturales. Crítica del humanismo abstracto. Madrid: Catarata.

Herrera, M. (2001). Formación inicial y profesionalización del magisterio. En S. Sandoval (comp.), La formación de educadores en Colombia. Geografías e imaginarios. Bogotá: UPN.

Hoyos, G. (2012). Ensayos para una teoría discursiva de la educación. Bogotá: Magisterio.

Instituto Interamericano de Derechos Humanos (2003). II Informe Interamericano de la Educación en Derechos Humanos: Desarrollo en el currículo y textos escolares. Recuperado de http://iidhwebserver.iidh.ed.cr/multic/UserFiles/Biblioteca/IIDH/3_2010/b011a7cb1540-49bc-a88d-ee727e77423f.pdf

Latorre, A. (2007). La investigación-acción. Conocer y cambiar la práctica educativa. Barcelona: Grao.

Mc Laren, P. (2005). La vida en las escuelas. México: Siglo XXI.

Magendzo, A. (2005). Educación en derechos humanos. Bogotá: Magisterio.

Martínez, M. (2008). Redes pedagógicas: la constitución del maestro como sujeto político. Bogotá: Magisterio.

Nuin, S. (2008). Dibujando fuera de los márgenes. Movimientos sociales en América Latina. Entrevista a Raúl Zibechi. Buenos Aires: La Crujía.

Plan Nacional de Educación en Derechos Humanos PLANEDH (2009). Bogotá: Defensoría del PuebloMinisterio de Educación Nacional-Vicepresidencia de la República.

República de Colombia. Ministerio de Educación Nacional. Decreto 1860 de 1994 (Reglamentario de la Ley 115 de 1994).

Ricœur, P. (1999). Lo justo. Madrid: Caparrós.

Sacavino, S. (2012). Democracia y educación en derechos humanos en América Latina. Bogotá: Desde Abajo.

Santos, B. (1998). De la mano de Alicia. Lo social y lo político en la posmodernidad. Bogotá: Siglo del Hombre.
Santos, B. (1998b). La globalización del derecho. Los nuevos caminos de la regulación y la emancipación, Bogotá: Universidad Nacional de Colombia.

Siede, I. (2007). La educación política. Ensayos sobre ética y ciudadanía en la escuela. Buenos Aires: Paidós.

Torres, A. (2004). Por una investigación desde el margen. En A. Jiménez y A. Torres (comp.), La práctica investigativa en ciencias sociales (pp. 63-80). Bogotá: UPN. 